\title{
Second EACR Conference on Cell Death in Cancer, Amsterdam, The Netherlands, 30 January-1 February 2014
}

\author{
V Jendrossek ${ }^{\star, 1}$ and S Fulda ${ }^{\star, 2}$
}

Cell Death and Differentiation (2014) 21, 1180-1182; doi:10.1038/cdd.2014.51; published online 25 April 2014

Second EACR Conference on Cell Death in Cancer, at De Rode Hoed, Amsterdam, The Netherlands, 30 January-1 February 2014

\begin{abstract}
Apoptotic and Non-Apoptotic Cell Death Pathways
Pascal Meier (London, UK) discussed caspase regulation in apoptotic and non-apoptotic signaling. Activation of initiator caspases is regulated via the assembly of multi-protein platforms that integrate cellular signals and recruit initiator caspases via their death-fold domain. To gain novel insights into the regulatory mechanisms that govern activation of initiator caspases, his lab used a proteomics-based approach. This strategy resulted in the identification of an evolutionarily conserved regulator of caspases that controls caspases in their apoptotic and non-apoptotic roles.

Martin Leverkus (Mannheim, Germany) reported on the importance of cFLIP for maintaining the integrity of the epidermis. He showed that mice constitutively lacking cFLIP in the epidermis die early during embryonic development. Abrogation of cFLIP in adult skin resulted in severe skin inflammation associated with caspase activation and apoptotic cell death that depends on autocrine production of inflammatory cytokines such as tumor necrosis factor-alpha $(\mathrm{TNF} \alpha)$. The clinical relevance of these findings was underscored by data showing that cFLIP protein was lost in the epidermis of patients with severe drug reactions associated with epidermal apoptosis.

Douglas Green (Memphis, TN, USA) discussed how caspases and receptor-interacting protein kinases (RIPK) control cell death and development, and introduced another level of complexity. He proposed a model in which two signals function to regulate RIPK1 activation. Signal 1 is considered to originate from the TNFR1, promotes apoptosis via Fas-associated protein with death domain (FADD)-caspase-8 and is inhibited by RIPK1. There is evidence showing that signal 2 is derived from cell surface toll-like receptors and interferons and can promote RIPK3 activation and necroptosis, while RIPK1 negatively regulates these events.
\end{abstract}

Peter Vandenabeele (Ghent, Belgium) reported on molecular mechanisms involved in the regulation and execution of necroptosis with a specific focus on mixed lineage kinase domain-like protein (MLKL). According to structure function analysis, the first four alpha helices at the $\mathrm{N}$-terminus of MLKL are critically required for its cytotoxic activity. This $\mathrm{N}$-terminal domain of MLKL was also necessary for the assembly of higher order structures as well as for the recruitment of MLKL to the plasma membrane.

Jannie Borst (Amsterdam, The Netherlands) highlighted the importance of ubiquitin-dependent degradation for the function of $\mathrm{Bcl}-2$ proteins. The lower potency of $\mathrm{Bcl}-\mathrm{B}, \mathrm{Bfl}-1$ and Mcl-1 was found to be due to their higher proteasomal turnover. By comparison, analysis of the quantitative interaction profile of all human pro-survival $\mathrm{Bcl}-2$ proteins, $\mathrm{BH} 3-$ only proteins, Bax and Bak revealed little selectivity, except for Noxa and Bad. These findings indicate that protein turnover rather than $\mathrm{BH} 3$ domain selectivity has a critical role in regulating the antiapoptotic activity of pro-survival $\mathrm{Bcl}-2$ proteins.

Patrick Mehlen (Lyon, France) introduced the concept that dependence receptors are tumor suppressors that limit tumor progression by inducing apoptosis of tumor cells under conditions when their ligands are not available or not accessible. Many aggressive cancers escape this form of programmed cell death by loss of dependence receptors or, alternatively, by autocrine expression of ligands such as netrin-1. A drug candidate that interferes on the interaction between netrin-1 and the corresponding dependence receptors DCC and UNC5H was shown to induce tumor cell death in vitro and regression of tumors and metastases in mice. The first phase I clinical trial in humans using an agent interfering between netrin-1 and its receptors is planned to start in mid 2015.

${ }^{1}$ Institute of Cell Biology (Cancer Research), University of Duisburg-Essen, Essen, Germany and ${ }^{2}$ Institute for Experimental Cancer Research in Pediatrics, Goethe-University, Frankfurt, Germany

${ }^{*}$ Corresponding author: V Jendrossek, Institute of Cell Biology (Cancer Research), University of Duisburg-Essen, Virchowstrasse 173, 45122 Essen, Germany. Tel: +49 201723 3380; Fax: +49 201723 5904; E-mail: Verena.Jendrossek@uni-due.de

or S Fulda, Institute for Experimental Cancer Research in Pediatrics, Goethe-University, Komturstrasse 3a, 60528 Frankfurt, Germany. Tel: +49 6967866557 ; Fax: +4969678 6659157; E-mail: simone.fulda@kgu.de 


\section{Targeting Cell Death Pathways in Cancer}

Marja Jäättelä (Copenhagen, Denmark) discussed the control of cancer cell survival and autophagy by sphingomyelin metabolism. She showed that acid sphingomyelinase (ASM) activity is required for maintaining lysosomal stability, autophagosome formation and multidrug resistance. Pharmacological inhibition of ASM by siramesine, an experimental anticancer agent, or by cationic amphiphilic drugs resulted in lysosomal cell death even in apoptosis- and multidrugresistant cells. The presented findings suggest that ASM inhibitors represent promising new cancer therapeutics even for therapy-resistant tumors.

Simone Fulda (Frankfurt, Germany) reported on recent discoveries showing that Obatoclax, a small-molecule inhibitor of antiapoptotic Bcl-2 proteins, triggers cell death via autophagy by stimulating the assembly of the necrosome on autophagosomal membranes, thereby connecting Obatoclaxstimulated autophagy to necroptosis. Blockade of autophagosome formation by silencing of Atg5 or Atg7 was found to abolish Obatoclax-mediated cell death, demonstrating that under these conditions autophagy is a cytodestructive mechanism.

Ruggero de Maria (Rome, Italy) introduced the use of a locked nucleic acid (LNA)-based anti-miR library to functionally screen cancer cells for putative oncogenic microRNAs ( $\mathrm{miR}$ ) that may be suited as selective therapeutic targets. He showed that miR-197 acts as a pro-survival factor in non-small cell lung cancer (NSCLC). Downregulation of miR-197 triggered p53-dependent apoptosis and prevented tumor growth in immunodeficient mice. The findings highlight a potential use of miR-197 as therapeutic target in NSCLC.

Verena Jendrossek (Essen, Germany) reported about the consequences of radiation-induced cell death for the adverse side effects of ionizing radiation in the lung. Using a murine model, she showed that radiation-induced local lung damage is associated with vascular dysfunction and increased seeding and growth of lung metastases from circulating tumor cells. She also demonstrated that functional impairment of the blood-air barrier as well as metastasis formation from circulating tumor cells in previously irradiated lung tissues were counteracted by adoptive transfer of mesenchymal stem cells. These data suggest that therapeutic application of mesenchymal stem cells is a promising strategy to prevent unwanted adverse late effects caused by radiation-induced damage to normal tissues.

Jochen Prehn (Dublin, Ireland) presented data on new patient stratification tools for apoptosis sensitizers based on system level analysis of apoptosis deficiency in cancer. The group developed a computational tool named DR_MOMP that incorporates the network knowledge on processes controlling mitochondrial outer membrane permeabilization (MOMP), leading to caspase-dependent and -independent cell death. This computational tool was shown to be capable of predicting treatment response both in vitro using colon cancer cell lines and in a clinical setting for colorectal cancer patients receiving adjuvant or neoadjuvant therapy. In addition, DR_MOMP could serve to identify patients who may benefit from the addition of $\mathrm{Bcl}-2$ antagonists.

\section{Tumor Suppressor Gene Networks/Animal Models}

Scott Lowe (New York, NY, USA) highlighted the importance of combining cancer genomics, murine cancer models and RNA interference screening to identify and validate candidate cancer driver and maintenance genes in their specific genetic context. He showed that targeted disruption of the histone methyltransferase MLL3 only promotes tumor formation in the context of specific genetic changes, such as a p53-deficient background or loss of the transcription factor NF1. He further reported that by targeted downregulation of genes that are specifically upregulated in the context of mutant p53, his group succeeded to identify a novel role of mutant p53 for disease progression in pancreatic cancer.

Jan Paul Medema (Amsterdam, The Netherlands) discussed the crucial role of the morphogenetic pathways Notch and Wnt in stem cells of normal colon tissue and colon cancer. By using matrigel-based intestinal organoid cultures from normal and cancerous tissue, he demonstrated that Notch signaling regulates self-renewal and lineage determination in both normal and transformed tissues, and that the Wnt pathway - despite activating mutations displays heterogeneous activity patterns in all disease stages. Finally, he reported that decreased mitochondrial priming contributes to chemoresistance of cancer stem cells due to upregulation of antiapoptotic $\mathrm{Bcl}-\mathrm{xL}$, which could be overcome by $\mathrm{BH} 3$ mimetics.

To gain insight into the contribution of lymphangiogenesis to metastatic spread and drug response the group of Marisol Soengas (Madrid, Spain) developed a lymphoreporter knockin mouse expressing enhanced green fluorescent protein (EGFP) luciferase in cells with activated VEGFR3, a classical marker of lymphangiogenesis. She showed that imaging of systemic VEGFR3 activation in lymph nodes shortly after tumor cell injection allows the identification of genes involved in metastasis and evaluation of the therapeutic potential of drugs interfering with the metastatic process. Finally, she demonstrated that synthetic dsRNA-based nanoparticles counteract metastasis formation in melanoma-bearing mice through activation of macrophages and subsequent active tumor regression.

\section{Autophagy, Metabolism and Cancer}

Sharon Tooze (London, UK) introduced the basic mechanisms of autophagy signaling and provided new insights into the molecular machinery driving autophagosome formation. She first highlighted the role of WD-repeat protein interacting with phosphoinosides (WIPI)2, a homolog of the autophagy (Atg) protein Atg18, for the lipidation of light chain (LC)3 family members on autophagosomal membranes and the deregulation of WIPI proteins in human skin cancer. She then reported novel findings on the molecular composition and regulation of WIPI2-containing multi-protein complexes driving autophagosome formation.

Nathalie Mazure (Nice, France) reported on novel mechanisms of hypoxia-mediated resistance of tumor cells to anticancer drugs involving hypoxia-inducible factor (HIF)1-dependent generation of enlarged mitochondria and a C-terminal-truncated variant of the mitochondrial 
voltage-dependent anion channel-1 (VDAC1), VDAC1- $\triangle \mathrm{C}$. VDAC $1-\Delta C$ retained the ability to bind to antiapoptotic hexokinase, thereby allowing cancer cells to maintain adenosine triphosphate (ATP) levels and survival in hypoxia. Inhibition of mitochondrial fusion or silencing of VDAC1- $\Delta C$ restored sensitivity of hypoxic cancer cells to chemotherapyinduced apoptosis.

Gerry Melino (Rome, Italy and Leicester, UK) reported on the involvement of p73, a p53 family member, in senescence and metabolism, and provided insight into several newly identified transcriptional p73 targets. He showed that p73 knockout mice show an early spontaneous aging phenotype (for example, alopecia, epidermal thinning, osteoporosis) at 14 months of age, indicating a transactivation-proficient (TA)p73-dependent protection against aging. TAp73-dependent protection involved regulation of mitochondrial activity and cellular accumulation of reactive oxygen species (ROS). He also presented in vitro data revealing the ability of TAp73 to drive the expression of glutaminase type 2 , thereby regulating cellular levels of glutamate, serine and glutathione. Altogether, these data demonstrate that TAp73-null cells have defects in the glutamine/serine metabolism and mitochondrial function affecting cellular redox balance and ROS defense.
Tak Mak (Toronto, ON, Canada) introduced the concept of targeting the consequences of oncogenic signaling in cancer such as alterations in cell metabolism and cellular redox state. He discussed the dual role of ROS in cancer development and in therapy response, and highlighted mechanisms that allow cancer cells to detoxify high ROS levels or to adapt to chronic oxidative stress. Moreover, he provided evidence for a role of estrogen-dependent regulation of ROS in the increased risk of breast cancer (BRCA)1 carriers to specifically develop breast and ovarian cancer. These results underline that a better understanding of cancer cell-specific metabolic alterations can pave the way for the design of more efficient treatment concepts for cancer patients.

\section{Conflict of Interest}

The authors declare no conflict of interest.

Acknowledgements. We thank EACR for substantial financial and administrative support, including five Meeting Bursaries for young researchers and two Poster Prizes. In particular, we are grateful to Kathryn Wass and Tabitha Chetwynd for the excellent organization of all administrative aspects of the meeting in Amsterdam. Furthermore, we are indebted to Pascal Meier, Nathalie Mazure, Patrick Mehlen, Marisol Soengas and Jannie Borst for their help with the abstract review and in the award committee. Finally, we thank all speakers, poster presenters and delegates for their excellent contributions and discussions that built the success of the meeting. 